\title{
EPIDEMIOLOGIA DA CÁRIE DENTÁRIA EM CRIANÇAS PRÉ-ESCOLARES DE UMMUNICÍPIO DO NORDESTE BRASILEIRO
}

Érika Vanessa Serejo Costa ${ }^{1}$ Cibelly Aliny Siqueira Lima Freitas ${ }^{1}$

Francisco Rosemiro Guimarães Ximenes Neto ${ }^{1}$ Maria Adelane Monteiro da Silva ${ }^{1}$

Luciano Garcia Lourenção https://orcid.org/0000-0001-6845-1965 https://orcid.org/0000-0002-0585-5345 https://orcid.org/0000-0002-7905-9990 https://orcid.org/0000-0001-7579-2645 https://orcid.org/0000-0001-7579-2645

Objetivo: Verificar a prevalência de cáries em pré-escolares, por meio do índice ceo-d. Método: Estudo transversal, desenvolvido entre fevereiro e outubro de 2016, com 189 crianças pré-escolares, com idade de cinco anos. Os dados foram coletados por meio de exame clínico, sistematizados no Microsoft Excel $\AA^{\circ}$ e analisados com estatística descritiva segundo diretrizes do manual da Pesquisa SB-Brasil 2010. Resultados: Obteve-se índice ceo-d 4,48, com predominância de 90,4\% do componente cariado, 3,4\% extração indicada, 6,1\% obturado. Apenas 20,1\% da amostra obteve ceo-d = 0 (não apresentou lesões de cárie) e $68,7 \%$ nunca visitou o dentista até o momento da realização da pesquisa. Conclusão: A análise dos resultados dos componentes "c", "e" e "d" do índice ceo-d infere que a alta prevalência da doença cárie está relacionada a fatores diversos, como higiene oral precária, medo/ansiedade, dificuldade de acesso aos serviços de saúde bucal ou escassez destes para a faixa etária.

Descritores: Saúde da Criança; Saúde Bucal; Estudos Epidemiológicos; Cárie Dentária.

\section{EPIDEMIOLOGY OF DENTAL CARIES IN PRE-SCHOOL CHILDREN OF A MUNICIPALITY IN THE BRAZILIAN NORTHEAST}

Objective: To verify the prevalence of caries disease in preschoolers using the ceo-d index. Method: Cross-sectional study, developed between February and October 2016, with 189 preschoolers with age of five years. The data were collected through clinical examination, systematized in Microsoft Excel ${ }^{\circledR}$ and analyzed with descriptive statistics according to guidelines of SB-Brazil 2010 Research Manual. Results: The ceo-d index was 4.48, with a predominance of $90.4 \%$ of the carious component, $3.4 \%$ indicated extraction, and $6.1 \%$ obturated. Only $20.1 \%$ of the sample obtained ceo- $d=0$ (did not present caries lesions) and $68.7 \%$ never visited the dentist until the moment of the research. Conclusion: The analysis of the results of the components "c", "e" and " $d$ " of the ceo-d index infers that the high prevalence of caries disease is related to diverse factors such as poor oral hygiene, fear/anxiety, difficulty accessing health services or shortage of these for the age group.

Descriptors: Child Health; Oral Health; Epidemiological Studies; Dental Caries.

\section{EPIDEMIOLOGÍA DE LA CARIE DENTAL EN NIÑOS EN EDAD PREESCOLAR EN UN MUNICIPIO DEL NORESTE DE BRASIL}

Objetivo: Verificar la prevalencia de caries en los niños en edad pré-escolar, por medio del índice ceo-d. Metodo: Estudio transversal, desarrollado entre febrero y octubre de 2016, con 189 niños de cinco años. Los datos fueron recolectados por medio de examen clínico, sistematizados en Microsoft Excel@ y analizados con estadísticas descriptivas de acuerdo con las directrices del Manual de Investigación SB-Brasil 2010. Resultados: Se obtuvo un índice ceo-d 4,48, con predominancia de 90,4\% del componente cariado, 3,4\% extracción indicada, 6,1\% obturado. Solamente 20,1\% de la muestra obtuvo ceo-d = 0 (no presentó lesiones de caries) y $68,7 \%$ nunca visitó al dentista hasta el momento de la realización de la investigación. Conclusión: El análisis de los resultados de los componentes "c", "e" y "d" del índice ceo-d, indica que la alta prevalencia de la caries está relacionada a factores diversos como higiene oral precaria, miedo/ansiedad, dificultad de acceso a los servicios de salud bucal o escasez de estos para esta franja de edad.

Descriptores: Salud del Niño, Salud Bucal; Estudios Epidemiológicos; Caries Dental.

IUniversidade Estadual Vale do Acaraú (UVA) Sobral, CE.

${ }^{2}$ Universidade Federal do Rio Grande (FURG) Rio Grande, RS.

Autor Correspondente: Francisco Rosemiro Guimarães Ximenes Neto - Email: rosemironeto@gmail.com

Recebido: 26/03/2020 - Aceito: 30/03/2020 


\section{INTRODUÇÃo}

A cárie dentária é um dos agravos bucais mais prevalentes do mundo, sendo considerada pela Organização Mundial da Saúde (OMS) um grave problema de Saúde Pública, que afeta sujeitos de todas as idades, cujo diagnóstico precoce se torna cada vez mais importante para dar subsídios ao estabelecimento de medidas preventivas adequadas. Na infância, a cárie é uma doença crônica comum, cuja etiologia multifatorial pode associar-se a fatores biológicos, comportamentais, socioeconômicos e de acesso aos serviços de saúde bucal.(1-4)

Além disso, as condições de saúde bucal se constituem em fatores de grande interferência na qualidade de vida dos sujeitos, impactando negativamente em todas as dimensões, seja física, funcional, nutricional e até mesmo psicossocial. A literatura mostra a influência marcante dos fatores sociais, econômicos e culturais na distribuição desigual dos problemas de saúde bucal na população, com maior prevalência de doenças na população com menos assistência social e sanitária. ${ }^{(4,5-7)}$

Em crianças com idade pré-escolar - até os seis anos de idade -, a cárie é conceituada pela American Academy of Pediatric Dentistry (AAPD) como Cárie Precoce da Infância (CPI), e definida como a presença de um ou mais dentes decíduos com cárie (cavitada ou não cavitada), restaurados ou perdidos devido a cárie. ${ }^{(8)}$

O índice ceo-d possibilita a expressão quantitativa de prevalência da cárie para a dentição decídua, medindo o percentual de crianças com dentes decíduos cariados (c), com extração indicada ou perdidos devido à cárie (e) e obturados (o), em determinado período e espaço geográfico. Segundo a Federation Dentaire Internationale (FDI), a idade de cinco anos é adotada internacionalmente como parâmetro para uso do indicador, o qual é utilizado para analisar variações geográficas e temporais que demandem estudos especiais, bem como subsidiar os processos de planejamento, gestão, avaliação e implementação de ações, visando à melhoria da saúde bucal nesta faixa etária, o que resultará em reflexos positivos também para a dentição permanente desta população. ${ }^{(4,9-10)}$

No Brasil, os modelos de atenção à saúde bucal, até o final do Século XX, eram excludentes e impactaram negativamente nos indicadores epidemiológicos e de acesso aos serviços básicos de saúde. A baixa cobertura em saúde bucal originou uma geração de desassistidos, com mutilação parcial ou total.(11)

Resultados do índice de cárie ceo-d nas últimas Pesquisas Nacionais de Saúde Bucal, realizadas em 2010 e 2003 , desenham o panorama epidemiológico dessa desassistência. De acordo com os dados do Ministério da Saúde, em 2010, na dentição decídua, o ataque de cárie em crianças de cin- co anos foi, em média, de 2,43 dentes; desses, menos de $20 \%$ estavam tratados no momento em que foram realizados os exames epidemiológicos. Em 2003, a média nessa idade era de 2,8 dentes, uma redução pequena para um período de sete anos. ${ }^{(12-13)}$

Ante o exposto, este estudo objetivou verificar a prevalência de cáries em pré-escolares, por meio do índice ceo-d.

\section{MÉTODO}

\section{Tipo de Estudo}

Trata-se de um estudo epidemiológico transversal, do tipo estudo de caso.

\section{Local do estudo}

O estudo foi desenvolvido no município de Tianguá - Ceará, Brasil, durante o período de fevereiro a outubro de 2016. O município possui uma população estimada de 73.468 habitantes, é polo e sede da microrregião da saúde da Serra da Ibiapaba, 13a - Coordenadoria Regional de Saúde (CRES), pertencente à Macrorregião da Saúde de Sobral.

\section{Participantes do Estudo}

A população investigada consistiu em crianças pré-escolares, com idade de cinco anos, residentes no município de Tianguá, que atenderam os seguintes critérios de inclusão: crianças na idade de cinco anos; estar cadastrado nas Unidades de Saúde da Família (USF) participantes, que assentiram participar da pesquisa, após o consentimento dos responsáveis, totalizando 300 crianças.

Utilizou-se o referencial da OMS, que considera a idade de cinco anos de importante interesse em relação aos níveis de doenças bucais na dentição decídua, uma vez que estas podem exibir mudanças em um período de tempo menor do que a dentição permanente em outras idades-índice, além de ser usada internacionalmente para aferição do ataque de cárie em dentes deciduos. ${ }^{(14)}$

A amostra da pesquisa foi obtida utilizando técnicas de amostragem probabilística proporcional estratificada, de acordo com a população de cada USF, considerando erro amostral de 5\%. A base do cálculo da amostra correspondeu a 171 crianças e, devido arredondamento de frações para números inteiros no número de participantes por USF, determinou-se amostra mínima de 176 crianças.

\section{Procedimentos de Coleta de Dados}

Como a pesquisa foi desenvolvida no momento de transição do Sistema de Informação da Atenção Básica (SIAB) para e o e-SUS, foi necessário buscar dados primários de modo individualizado em cada USF, investigando junto a cada Agente 
Comunitário de Saúde (ACS) a quantidade de crianças cadastradas em suas respectivas microáreas. Por meio da articulação com o enfermeiro gerente das USF, os ACS organizaram suas demandas e agendaram dias específicos para a coleta de dados. Nos casos em que a articulação gerencial não foi efetiva ou o ACS não era bem aceito pela comunidade, os pesquisadores realizaram visitas domiciliares nos territórios, para a realização das avaliações bucais, até atingir a amostra planejada.

Após estratificação da amostra, a partir dos dados primários adquiridos por meio dos ACS, a coleta de dados foi realizada por uma única pesquisadora, sob iluminação artificial com lanterna em led ou refletor da cadeira odontológica.

Os instrumentais e materiais utilizados foram espelho clínico, máscara, luvas de procedimento, algodão em rolete e gazes estéreis. Os resultados foram registrados em planilhas, elaboradas especificamente para a pesquisa. A avaliação bucal foi realizada nas USF (com agendamento prévio), nas creches dos territórios e nos domicílios das famílias participantes, sendo contabilizado, para cada criança, o número de dentes cariados, dentes com extração indicada, dentes obturados, total de dentes decíduos e se era a primeira consulta de saúde bucal.

\section{Procedimentos de Análise dos Dados}

Após a coleta, os dados foram submetidos aos critérios para o diagnóstico dos componentes do índice ceo-d cariado, extração indicada e obturado, segundo manual da Pesquisa SB-Brasil 2010 ${ }^{(14)}$, considerando-se:

- Elemento dentário hígido: dentes decíduos sem evidência de cárie. Estágios iniciais da doença não foram considerados;

- Elemento dentário cariado (c): dentes decíduos cavitados ou com tecido amolecido na base, ou descoloração do esmalte, ou de parede, ou com uma restauração temporária (exceto ionômero de vidro);

- Elemento dentário com extração indicada (e): dente decíduo não está no período de esfoliação e tem indicação de extração por motivo de cárie e não por outras razões; e

- Elemento dentário obturado (o): dentes decíduos com uma ou mais restaurações definitivas, com cárie recorrente ou não.

Os dados obtidos foram tratados estatisticamente e apresentados de forma descritiva.

\section{Procedimentos Éticos}

O estudo respeitou os aspectos éticos e legais que regulamentam pesquisas envolvendo seres humanos, estabelecidos pela Resolução no 466/2012, do Conselho Nacional de
Saúde, sendo aprovado pelo Comitê de Ética em Pesquisa da Universidade Estadual do Acaraú (CEP/UVA), sob Parecer n의 1.434 .836 .

Antecedendo a coleta dos dados, foi solicitado ao pais ou responsáveis pelas crianças que concordaram participar da pesquisa, a assinatura do Termo de Consentimento Livre e Esclarecido, bem como do Termo de Assentimento da criança, para a realização da avaliação bucal.

\section{RESULTADOS}

Em virtude do arredondamento amostral na randomização por USF, a amostra final foi constituida por 189 crianças examinadas. Na Tabela 1 é apresentada a distribuição do número de microáreas, população (total de crianças com cinco anos de idade), amostra, número de crianças examinadas e o número de crianças que realizaram primeira consulta odontológica, segundo USF incluída no estudo. Observa-se que, em nove USF (90,0\%), mais da metade das crianças nunca haviam realizado consulta odontológica e, para 130 (68,8\%) crianças esta foi a primeira avaliação bucal realizada.

Tabela 1 - Distribuição do número de microáreas, população e amostra, número de crianças examinadas e de crianças que realizaram primeira consulta odontológica, segundo USF incluída no estudo.

\begin{tabular}{|c|c|c|c|c|c|c|c|}
\hline \multirow{2}{*}{ USF } & \multicolumn{2}{|c|}{ Microáreas } & \multirow{2}{*}{$\begin{array}{c}\begin{array}{c}\text { Po- } \\
\text { pula- } \\
\text { ção }\end{array} \\
\text { n }\end{array}$} & \multirow{2}{*}{$\begin{array}{c}\begin{array}{c}\text { Amos- } \\
\text { tra } \\
(\mathrm{e}=5 \%)\end{array} \\
n\end{array}$} & \multirow{2}{*}{$\begin{array}{c}\begin{array}{c}\text { Crian- } \\
\text { ças } \\
\text { Exami- } \\
\text { nadas }\end{array} \\
\mathbf{n}\end{array}$} & \multicolumn{2}{|c|}{ 1a Consulta } \\
\hline & $\mathbf{n}$ & $\%$ & & & & $\mathbf{n}$ & $\%$ \\
\hline Ceasa & 5 & 10,9 & 44 & 25,1 & 26 & 22 & 84,6 \\
\hline $\begin{array}{l}\text { Centro de } \\
\text { Nutrição }\end{array}$ & 4 & 8,7 & 54 & 30,8 & 31 & 20 & 64,5 \\
\hline $\begin{array}{l}\text { Centro de } \\
\text { Saúde I }\end{array}$ & 6 & 13,0 & 22 & 12,5 & 13 & 10 & 76,9 \\
\hline Córrego & 6 & 13,0 & 44 & 25,1 & 31 & 25 & 80,6 \\
\hline Frecheiras & 4 & 8,7 & 15 & 8,6 & 9 & 8 & 88,8 \\
\hline $\begin{array}{l}\text { Governador } \\
\text { Ferraz }\end{array}$ & 4 & 8,7 & 31 & 17,7 & 25 & 14 & 56,0 \\
\hline $\begin{array}{l}\text { Isabel } \\
\text { Nogueira }\end{array}$ & 3 & 6,5 & 11 & 6,3 & 7 & 2 & 28,5 \\
\hline Joana Urias & 5 & 10,9 & 36 & 20,5 & 21 & 12 & 57,1 \\
\hline Planalto & 5 & 10,9 & 21 & 12,0 & 13 & 10 & 76,9 \\
\hline Rodoviária & 4 & 8,7 & 22 & 12,5 & 13 & 7 & 53,8 \\
\hline Total & 46 & 100 & 300 & 171 & 189 & 130 & 68,8 \\
\hline
\end{tabular}


Os resultados do índice ceo-d das crianças atingiram o valor médio de 4,48, com 90,4\% de predominância do componente cariado (c) e oscilando entre ceo-d mínimo de 3,46 na USF Planalto e máximo de 5,42 na USF Isabel Nogueira.

A comparação do índice ceo-d obtido em Tianguá com resultados da Pesquisa SB - Brasil 2010 de Fortaleza, de municipios do interior do Nordeste e do Brasil, evidenciou que o municipio de Tinguá possui prevalência de cárie superior ao das outras localidades (Figura 1)

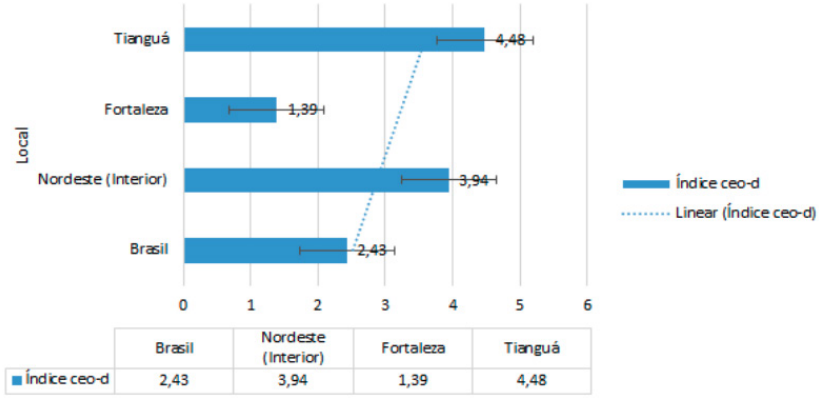

Figura 1 - Comparação do índice ceo-d obtido em Tianguá com resultados da Pesquisa SB - Brasil 2010 de Fortaleza, de municípios do interior do Nordeste e do Brasil.

Conforme mostra a Tabela, o número de crianças sem cáries - ceo-d = 0 -, bem como o índice ceo-d encontrado em todas as USF são superiores à média nacional.
A Figura 2 mostra a distribuição do percentual de crianças sem cáries (ceo- $d=0$ ) ou com ceo- $d \geq 1$. Observa-se que, em todas as USF, o percentual de crianças sem cáries está abaixo de $30,0 \%$, sendo a média geral de crianças livres de cárie de $20,1 \%$.

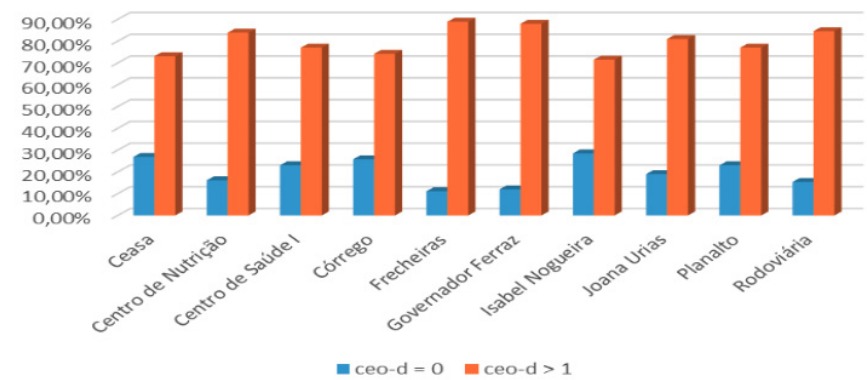

Figura 2 - distribuição do percentual de crianças sem cáries (ceo-d = 0) ou com ceo- $d \geq 1$.

\section{DISCUSSÃO}

A análise dos percentuais de cada componente do índice ceo-d evidencia que, maiores valores no componente "o" (elemento dentário obturado) indicam maior probabilidade de acesso da criança aos serviços de saúde bucal, diferentemente dos componentes "c" (elemento dentário cariado) e "e" (elemento dentário com extração indicada), cuja história presente da doença sugere ausência/dificuldade de acesso.(15) Nesse contexto, os altos valores apontados neste estudo, para o componente elemento dentário cariado, evidenciam dificuldade de acesso e possível escassez de serviços de saúde bucal no município em estudo, direcionados à esta população,

Tabela 2 - Distribuição do número de crianças sem cáries, em cada componente do índice ceo-d e valores do índice ceo-d, por USF.

\begin{tabular}{|c|c|c|c|c|c|c|c|c|c|c|c|c|}
\hline \multirow{3}{*}{ Unidade de Saúde } & \multirow{2}{*}{\multicolumn{2}{|c|}{ Amostra }} & \multirow{2}{*}{\multicolumn{2}{|c|}{$\begin{array}{l}\text { Crianças } \\
\text { Sem Cárie }\end{array}$}} & \multicolumn{7}{|c|}{ Elementos Dentários } & \multirow{3}{*}{$\begin{array}{l}\text { Indice } \\
\text { ceo-d }\end{array}$} \\
\hline & & & & & \multicolumn{2}{|c|}{$c^{*}$} & \multicolumn{2}{|c|}{$\mathrm{e}^{* *}$} & \multicolumn{2}{|c|}{$0^{* * *}$} & \multirow{2}{*}{ Total } & \\
\hline & $\mathbf{n}$ & $\%$ & $\mathbf{n}$ & $\%$ & $\mathbf{n}$ & $\%$ & $\mathbf{n}$ & $\%$ & n & $\%$ & & \\
\hline Córrego & 31 & 16,4 & 8 & 25,8 & 128 & 92,0 & 6 & 4,3 & 5 & 3,5 & 139 & 4,48 \\
\hline Gov. Ferraz & 25 & 13,2 & 3 & 12,0 & 104 & 85,9 & 12 & 9,9 & 5 & 4,1 & 121 & 4,84 \\
\hline Joana Urias & 21 & 11,1 & 4 & 19,0 & 91 & 90,0 & - & - & 10 & 9,9 & 101 & 4,80 \\
\hline Centro Saúde I & 13 & 6,8 & 3 & 23,0 & 40 & 86,9 & 2 & 4,3 & 4 & 8,6 & 46 & 3,53 \\
\hline Frecheiras & 9 & 4,7 & 1 & 11,1 & 40 & 97,5 & - & - & 1 & 2,5 & 41 & 4,55 \\
\hline Isabel Nogueira & 7 & 3,7 & 2 & 28,5 & 36 & 94,7 & 2 & 5,2 & - & - & 38 & 5,42 \\
\hline Total & 189 & 100 & 38 & 20,1 & 766 & 90,4 & 29 & 3,4 & 52 & 6,1 & 847 & 4,48 \\
\hline
\end{tabular}


fato que é reforçado pelo alto percentual de crianças que, aos cinco anos de idade, ainda não haviam realizado nenhuma consulta odontológica.

O importante aumento do índice de cáries nas crianças estudadas, em relação a outras regiões brasileiras aponta a necessidade de estratégias de intervenção no município. De acordo com os resultados obtidos no estudo, o índice ceo-d do município é três vezes maior que o da capital do estado e quase $20 \%$ maior do que o apresentado por municípios do interior da região nordeste. Além disso, enquanto a Pesquisa SB-Brasil 2010, aponta que 46,6\% das crianças brasileiras com cinco anos de idade estão livres de cárie ${ }^{(15)}$, este estudo evidenciou que apenas $20,1 \%$ das crianças do município não apresentaram lesões de cárie, ou seja, percentual muito inferior ao observado no Brasil.

Estudos realizados na região nordeste apontam índices ceo-d inferiores ao encontrado na presente pesquisa, reforçando a baixa cobertura e má qualidade das ações de saúde bucal no município avaliado.

Em Brejo dos Santos, Paraiba, estudo com pré-escolares de quatro e cinco anos de idade identificou experiência de cárie $50,9 \%$ e índice ceo-d médio de 1,15. Mesmo com esses valores inferiores aos observados nesta pesquisa, os autores consideraram o índice de cárie dentária elevado na população pre-escolar. ${ }^{16}$ Corroborando, estudos realizados em Fortaleza, Ceará, apontam ceo-d para crianças na faixa etária de três a seis anos variando entre 1,62 e 3,97,(17-18) reforçando os resultados da Pesquisa SB-Brasil 2010 no estado do Ceará, que mostrou menor prevalência de cáries na infância, na capital do que no interior. ${ }^{(12)}$

Outros estudos realizados em municípios de outras regiões do Brasil demonstraram valores inferiores aos observados nesse estudo, tais como Araçatuba, São Paulo, cujo ceo-d médio foi de 1,88 , sendo composto por $78 \%$ de dentes cariados, $21 \%$ de dentes restaurados e $1 \%$ de dentes com extração indicada; (19) Mineiros, Goiás, onde o ceo-d médio foi de 1,77, com predominância do componente cariado; ${ }^{20}$ e Montes Claros, Minas Gerais, ceo-d 1,17.(21)

O elevado percentual observado no componente C (dente cariado), associado ao baixo valor do componente $\mathrm{O}$ (dente obturado) indica insuficiência na provisão de cuidados odontológicos (muitas cáries vs. poucas restaurações), que pode ser decorrente da descontinuidade do programa de atenção à saúde bucal no município. Este cenário indica a necessidade de implementação de ações de atenção à saúde bucal eficazes, que superem o subdimensionamento dos recursos e a descontinuidade da assistência, enfatizando a dimensão da prevenção como estratégia de atenção, e investindo em pesquisas que possibilitem gerar conhecimentos capazes de contribuir para melhorar a situação atual.(22)

Nesse contexto, os resultados do presente estudo são fundamentais para que os profissionais da saúde discutam, planejem, implantem, implementem e avaliem as ações de saúde bucal que, muitas vezes, são direcionadas aos escolares, deixando os pré-escolares em segundo plano.(19) $A$ literatura brasileira destaca uma certa tendência de crescimento da prevalência e gravidade da cárie no país, com o avançar da idade, quando os resultados de um ceo- $d$ baixo podem evoluir para um cpo-d alto. ${ }^{(12-13,23)}$

Desta forma, a dificuldade de acesso aos serviços de saúde bucal, associada às maiores taxas de dentes cariados, na composição do índice ceo-d, justifica a importância de direcionar ações de atenção à saúde bucal nessa faixa etária. Nesse contexto, além de considerar a necessidade de mudar comportamentos de cuidados com saúde bucal na criança, é imprescindível que os gestores e os profissionais da saúde estejam atentos aos aspectos culturais que, muitas vezes, pressupõe o entendimento dos pais e educadores, de que os dentes decíduos serão substituídos, sendo, por este motivo, desvalorizados. ${ }^{(24)}$

Estudo com crianças institucionalizadas e não institucionalizadas com ceo-d elevado aponta a ausência do cirurgião-dentista, como maior fator de risco para o aparecimento de cáries nas crianças institucionalizadas, apesar de as crianças não institucionalizadas, que dispunham de cuidados de saúde bucal, também terem apresentado elevado índice de cáries. Para os autores, os resultados denotam a necessidade de implantação e/ou manutenção de assistência à saúde bucal de modo efetivo, seja nas escolas ou em abrigos, para que os índices de cáries possam ser reduzidos em médio e longo prazo. ${ }^{(25)}$

Não obstante, ressalta-se a fragilidade do indicador relacionado à primeira consulta de saúde bucal, que mostrou-se elevado no presente estudo. Apesar de medir a porcentagem da população que teve acesso aos serviços públicos de saúde bucal, muitas vezes esse valor é superestimado, incorporando os atendimentos de emergência, realizados na primeira consulta. Do ponto de visto odontológico e da saúde pública, esses atendimentos não representam intervenção para a adequada recuperação e controle das doenças bucais, pois o atendimento é pontual e, para os serviços, não demonstra resolutividade, pois o cliente não retorna para completar o tratamento após a resolução imediata do problema. ${ }^{(26)}$

É o que se confirma na análise da relação entre os valores do ceo-d em cada USF e as respectivas percentagens de primeira consulta encontradas no presente estudo. Independentemente do percentual de crianças em primeira consulta ser alto ou baixo, o índice ceo-d foi elevado, o que sugere não haver relação direta entre tais indicadores neste estudo.

Estudo realizado no interior do estado de São Paulo corrobora esses resultados ao evidenciar que um método preventivo isolado não é suficiente para assegurar baixos índices de cárie dentária. A alta prevalência da doença pode ser proveniente de fatores diversos, como a deficiência ou ausência 
dos procedimentos de higienização oral, e não apenas da dificuldade de acesso ao serviço de saúde bucal. Para os autores, a saúde bucal da criança pode estar mais relacionada aos conhecimentos de saúde bucal de seus familiares, o que exige que as ações de saúde bucal também atinjam os cuidadores, especialmente aquelas voltadas para a prevenção de riscos, de cáries e de mutilações precoces, as de educação em saúde e de promoção da saúde bucal.(19)

No entanto, esses resultados não excluem a importância de garantir acesso aos serviços de saúde bucal, imprescindíveis na busca de melhores condições de vida. Para se ter ideia, no Brasil, as crianças realizam a primeira visita ao dentista entre os quatro e cinco anos de vida, enquanto o recomendado é que o início dos atendimentos de saúde bucal ocorra entre seis meses e um ano de idade, demonstrando que a busca por serviços de saúde bucal ocorre muito tardiamente. ${ }^{(27)}$

Há um entendimento cultural de que o cirurgião-dentista cuida dos dentes quando estão "doentes". Neste contexto, a presença de bebês no consultório de saúde bucal é reduzida e, muitas vezes, quase inexistente em muitas unidades de saúde. Além disso, a não universalização dos serviços de saúde bucal na Atenção Primária à Saúde (APS) exige que os poucos serviços existentes deem conta das urgências odontológicas e dos problemas bucais dos adultos, que interferem na sua capacidade produtiva, comprometendo a implementação das ações de promoção da saúde bucal, bem como a ampliação do atendimento para as crianças. ${ }^{(28-30)}$

Assim, compreende-se que a garantia de acesso universal à atenção odontológica na Estratégia Saúde da Família (ESF), por meio da ampliação das equipes, de investimentos na qualificação da gestão e implementação de práticas de saúde baseadas em evidência contribui para a melhoria das condições de saúde bucal da população brasileira. Além disso, assegurar o desenvolvimento de práticas promotoras de saúde bucal, de ações de prevenção de doenças e recuperação da saúde bucal, que corroborará com a melhoria das condições de saúde e resgaste social de populações vulneráveis e carentes..$^{30-31}$

Além do acesso às ações de saúde bucal, a educação em saúde, com orientações aos pais e estímulo à prevenção da cárie, com a garantia do acesso aos insumos para escovação e higienização bucal são fundamentais. Neste sentido, a educação em saúde passar a ser um movimento instituinte e "eixo estruturante para a transformação da realidade".(29-30)

\section{Limitações do Estudo}

O estudo apresenta limitações importantes no que se refere a população e a amostra, uma vez que o período da coleta de dados coincidiu com a transição do Sistema de Informação da Atenção Básica (SIAB) para e o e-SUS e o processo de identificação das crianças se deu pelo contato com a Equipe da ESF, especialmente os agentes comunitários de saúde e não diretamente no sistema.

Outro aspecto que deve ser considerado é o fato do cenário da pesquisa compreender apenas um município da 13a Coordenadoria Regional de Saúde (CRES) da Macrorregião de Saúde de Sobral, Ceará.

\section{Contribuições para a Prática}

Considerando que o conhecimento da distribuição das doenças em uma população é de extrema relevância para o delineamento de ações e a organização dos serviços de saúde, o estudo contribui com informações importantes para o direcionamento de políticas e ações de atenção à saúde bucal em pré-escolares, fornecendo subsídios para o planejamento e a efetivação de estratégias interventivas, principalmente no nível local da APS.

\section{CONCLUSÃO}

A identificação do perfil epidemiológico da cárie em crianças pré-escolares constituiu-se ferramenta chave para o reconhecimento real do problema na população estudada, premissa básica para que gestores e profissionais de saúde desenvolvam estratégias de planejamento, execução e avaliação de ações interventivas, aliadas à prática do cuidado promotor de saúde bucal, especialmente na puericultura, ainda fragmentada e restrita às ações pontuais na APS local.

Os resultados do estudo apontam que, após quase 20 anos de institucionalização da Política Brasileira de Saúde Bucal, a partir de Programas como o "Brasil Sorridente" e da ampliação da Equipe de Saúde Bucal (eSB) junto à ESF, os valores dos componentes C, E e D do índice ceo-d inferem que a alta prevalência de cáries está mais relacionada à dificuldade de acesso e escassez de serviços do que à não universalização da cobertura dos serviços de saúde bucal para a população do município estudado, o que se assemelha diversos municipios brasileiros.

Inferências desta natureza são importantes para a análise de causa, entretanto, constatou-se a influência de outros fatores, como deficiência ou ausência dos procedimentos de higiene oral; medo e ansiedade, que permeiam a primeira visita ao cirurgião-dentista, para realização do exame de saúde bucal, e justificam a necessidade de implantação de ações de saúde bucal aos pré-escolares e seus cuidadores, especialmente as direcionadas à educação e promoção da saúde bucal.

\section{CONTRIBUIÇÃO DOS AUTORES:}

ÉVS Costa e CASL Freitas participaram da concepção e delineamento do estudo, redação e revisão do conteúdo intelectual até a versão final do manuscrito. FRG Ximenes Neto e MAA Monteiro participaram do delineamento, da redação e revisão do conteúdo intelectual até a versão final do manuscrito. LG Lourenção participou da revisão do conteúdo intelectual até a versão final do manuscrito. 


\section{REFERÊNCIAS}

1. Melo P. Azevedo A, Henriques M. Cárie dentária - a doença antes da cavidade. Acta Pediatr Port. 2008 [cited 2020 Mar 24]: 39(6):253-9. Available from: http://actapediatrica.spp.pt/article/view/4616/3453

2. Cangussu MC, Johelle Passos-Soares J, Cabral MB. Necessidades e problemas de saúde bucal no Brasil e tendências para as politicas de saúde. In: Chaves SCL (org.). Política de saúde bucal no Brasil: teoria e prática. Salvador: EDUFBA, 2016.

3. Januário BS, Figueiredo MC, Faustino-Silva DD. Avaliação de protocolos de manejo de cárie em crianças de 0 a 3 anos de idade baseada no risco. Stomatos. 2017 [cited 2020 Mar 24]; 23(45):14-25. Available from: http://www.periodicos.ulbra.br/index.php/stomatos/article/ view/3133

4. Nunes VH, Perosa GB. Cárie dentária em crianças de 5 anos: fatores sociodemográficos, lócus de controle e atitudes parentais. Ciênc. saúde coletiva. 2017 [cited 2020 Mar 24]; 22(1):191-200. DOI: https://doi.org/10.1590/1413-81232017221.13582015

5. Pereira AL. Influência da condição de saúde bucal na qualidade de vida dos individuos. [Trabalho de Conclusão de Curso]. Campos Gerais: Universidade Federal de Minas Gerais, Faculdade de Odontologia; 2010. [cited 2020 Mar 24]. Available from: https://www.nescon.medicina. ufmg.br/biblioteca/imagem/0986.pdf.

6. Lawder JA, Matos M, Souza JB, Freire MC. Impact of oral condition on the quality of life of homeless people. Rev. saúde pública. 2019 [cited 2020 Mar 24]; 53:22. DOI: https://doi.org/10.11606/S1518-8787.2019053000718

7. Souza RL, Guimarães ARD. Influência da condição bucal na qualidade de vida de adolescentes: uma revisão integrativa. Anais SEMIC. 2017 [cited 2020 Mar 24]; 21:1941. Available from: http://periodicos.uefs.br/ojs/index.php/semic/article/view/2556/1941.

8. Oliveira DA. Cárie dentária na infância - exposição precoce a fatores de risco. [Trabalho de Conclusão de Curso]. Porto: Universidade do Porto, Faculdade de Medicina Dentária; 2017. [cited 2020 Mar 24]. Available from: https://repositorio-aberto.up.pt/bitstream/10216/107228/2/211841.pdf.

9. Scalioni FAR, Figueiredo SR, Curcio WB, Alves RT, Leite ICG, Ribeiro RA. Hábitos de dieta e Cárie Precoce da Infância em crianças atendidas em Faculdade de Odontologia Brasileira. Pesq Bras Odontoped Clin Integr. 2012 [cited 2020 Mar 24]; 12(3):399-404. Available from: http://revista.uepb.edu.br/index.php/pboci/article/viewFile/1352/880.

10. Departamento de Informática do SUS - DATASUS (Brasil). Proporção de crianças de 5-6 anos de idade com indice ceo-d igual a 0 - G.18 - 2011. [cited 2020 Mar 24]. Available from: http://fichas.ripsa.org.br/2011/g-18/.

11. Gonçalves CÂA, Vazquez FL, Ambrosano GMB, Mialhe FL, Pereira AC, Sarracini KLM et al. Estratégias para o enfrentamento do absenteismo em consultas odontológicas nas Unidades de Saúde da Familia de um município de grande porte: uma pesquisa-ação. Ciênc. saúde coletiva. 2015 [cited 2020 Mar 24]; 20(2):449-60. DOI: https://doi.org/10.1590/1413-81232015202.00702014

12. Ministério da Saúde (BR). Projeto SB-Brasil 2010: Pesquisa Nacional de Saúde Bucal - Resultados Principais. Brasilia; 2012. [cited 2020 Mar 24]. Available from: http://bvsms.saude.gov.br/bvs/publicacoes/pesquisa_nacional_saude_bucal.pdf.

13. Ministério da Saúde (BR). Projeto SB Brasil 2003: Condições de saúde bucal da população brasileira 2002-2003 - Resultados Principais. Brasilia; 2004. [cited 2020 Mar 24]. Available from: http://bvsms.saude.gov.br/bvs/publicacoes/condicoes_saude_bucal.pdf.

14. Ministério da Saúde (BR). Pesquisa SB-Brasil 2010 - manual da equipe de campo. Brasilia; 2009. [cited 2020 Mar 24]. Available from: http://bvsms.saude.gov.br/bvs/publicacoes/sbbrasil_2010_pesquisa_nacional_saude_bucal_manual_equipe_campo.pdf.

15. Müller IB, Castilhos ED, Camargo MBJ, Gonçalves H. Experiência de cárie e utilização do serviço público odontológico por escolares: estudo descritivo em Arroio do Padre, Rio Grande do Sul, 2013. Epidemiol. Serv. Saúde. 2015 [cited 2020 Mar 25]: $24(4): 759-70$. DOI: https:// doi.org/10.5123/S1679-49742015000400018

16. Silva DDP, Camelo CCA, Carvalho MMP. Paredes SO. Experiência de cárie dentária e fatores associados em crianças pré-escolares. Arq Odontol. 2018 [cited 2020 Mar 25]; 54:e04. Available from: https://periodicos.ufmg.br/index.php/arquivosemodontologia/article/view/3759. 17. Almeida ACEB. Saúde bucal em crianças com idade indice de cinco anos pertencentes a familias em situação de pobreza beneficiadas 
pelo Programa Bolsa Familia. [Dissertação]. Fortaleza: Universidade Federal do Ceará, Faculdade de Farmácia, Odontologia e Enfermagem: 2014. [cited 2020 Mar 24]. Available from: http://www.repositorio.ufc.br/handle/riufc/8950

18. Vieira APGF, Karbage JP. Impacto da atenção odontológica na saúde bucal de crianças. RBPS. 2011 [cited 2020 Mar 24]: 24(1):10-15. Available from: http://periodicos.unifor.br/RBPS/article/view/2045/2339.

19. Garbin CAS, Chiba FY, Garbin AJI, Arcieri RM. Prevalência de cárie dentária em pré-escolares de escolas de educação infantil de Araçatuba, São Paulo. Revista Odontológica de Araçatuba. 2011 [cited 2020 Mar 24]; 32(2):28-32. Available from: http://hdl.handle. net/11449/133382.

20. Basílio KB, Vilela MIM, Braga RRS, Oliveira DC, Carrijo MO. Perfil de saúde bucal dos pré-escolares de uma escola municipal do sudoeste goiano. Revista Saúde Multidisciplinar. 2015 [cited 2020 Mar 24]; 3:162-75. Available from: https://www.fampfaculdade.com. br/wp-content/uploads/2019/03/Art.-12-PERFIL-DE-SA\%C3\%9ADE-BUCAL-DOS-PR\%C3\%89-ESCOLARES-DE-UMA-ESCOLA-MUNICIPAL-DO-SUDOESTE-GOIANO.pdf.

21. Tonelli SO, Rodrigues LAM, Alencar AM, Rodrigues CAQ. Avaliação do impacto de fatores socioeconômicos e acesso aos serviços de saúde bucal na prevalência de cárie dentária em crianças. RFO UPF [online]. 2016 [cited 2020 Mar 24]; 21(2):172-7. Available from: http:// revodonto.bvsalud.org/pdf/rfo/v2ln2/a04v2ln2.pdf.

22. Narvai PC, Campos JNS, Coelho CC, Frazão P, Rodrigues DA, Lemos PN. Cárie dentária em povos do Parque Indigena do Xingu, Brasil, 2007 e 2013. Epidemiol. Serv. Saúde. 2018 [cited 2020 Mar 24]; 27(1):e20171725. DOI: http://dx.doi.org/10.5123/s1679-49742018000100005 23. Moraes SNS, Arsenian MB, Tucci R. Avaliação clínica e utilização do índice CPO-D/“ceo-d" em crianças da Escola Municipal José Carlos Porto-Paraty/RJ. J Health Sci Inst. 2014 [cited 2020 Mar 24]; 32(3):235-40. Available from: https://www.unip.br/presencial/comunicacao/publicacoes/ics/edicoes/2014/03_jul-set/V32_n3_2014_p235a240.pdf.

24. Souza KFM, Oliveira WBC. Prevalência de cárie dentária em estudantes de uma escola pública na cidade de Porto Velho/RO. [Trabalho de Conclusão de Curso]. Porto Velho: Centro Universitário São Lucas; 2019. [cited 2020 Mar 24]. Available from: http://repositorio.saolucas.edu.br:8080/xmlui/bitstream/handle/123456789/3433/Katiana\%20Fortuna\%20de\%20Menezes\%20de\%20Souza,\%20Wilasmar\%20 Batista\%20C\%C3\%A9sar\%20de\%200liveira\%20-\%20Preval\%C3\%AAncia\%20de\%20c\%C3\%Alrie\%20dent\%C3\%Alria\%20em\%20estudantes $\% 20$ de $\% 20$ uma\%20escola\%20p\%C3\%BAblica $\% 20$ na $\% 20$ cidade $\% 20$ de\%20Porto\%20Velho\%20RO.pdf?sequence=1.

25. Vieira APGF, Karbage JP. Impacto da atenção odontológica na saúde bucal de crianças. RBPS. 2011 [cited 2020 Mar 24]; 24(1):10-15. Available from: http://periodicos.unifor.br/RBPS/article/view/2045/2339.

26. Junqueira SR, Frias AC, Zilbovicius C, Araujo ME. Saúde bucal e uso dos serviços odontológicos em função do Índice de Necessidades em Saúde: São Paulo, 2008. Ciênc. saúde coletiva. 2012 [cited 2020 Mar 24]: 17(4):1015-24. DOI: http://dx.doi.org/10.1590/S141381232012000400023

27. Silva JM, Penha ES. Acesso a serviços odontológicos e perda dentária em crianças. Revista Rede de Cuidados em Saúde. 2015 [cited 2020 Mar 25]: 9(1):1-11. Available from: http://publicacoes.unigranrio.br/index.php/rcs/article/view/2405.

28. Silva MCB (org.). Saúde da criança: questões da prática assistencial para dentistas. São Luís: UNA-SUS/UFMA; 2014. [cited 2020 Mar 25]. Available from: https://ares.unasus.gov.br/acervo/handle/ARES/513.

29. Neves M, Giordani JMA, Hugo FN. Atenção primária à saúde bucal no Brasil: processo de trabalho das equipes de saúde bucal. Ciênc saúde coletiva. 2019 [cited 2020 Mar 25]: 24(5):1809-20. DOI: https://doi.org/10.1590/1413-81232018245.08892017

30. Silva JMA, Silva J, Batista B, Carmo A, Gadelha M, Andrade M et al. Dificuldades experienciadas pelos agentes comunitários de saúde na realização da educação em saúde. Enferm. Foco. 2019 [cited 2020 Mar 25]; 10(3):82-7. Available from: http://revista.cofen.gov.br/index. php/enfermagem/article/view/1818/577.

31. Ximenes Neto FRG, Vasconcelos FM, Chagas MIO, Cunha ICKO, Sampaio JJC, Silva RCC. Nascimento da dentição em crianças menores de um ano: análise do perfil, percepção e práticas maternas e suas implicações para a organização dos serviços de saúde. Biblioteca Lascasas. 2010 [cited 2020 Mar 25]; 6(1). Available from: http://www.index-f.com/lascasas/documentos/lc0506.pdf 